\title{
RESULTADOS DEL ESTUDIO “PROYECTO PARA DISEÑAR Y APLICAR LOS MECANISMOS DE SEGUIMIENTO Y EVALUACIÓN DE LA PRIMERA FASE DEL MODELO SECTORIAL DE FORMACIÓN MUSICAL EN EL MARCO DEL PROYECTO JORNADA ESCOLAR 40 HORAS EN BOGOTÁ, COLOMBIA"
}

\author{
Milton Eduardo Bermúdez Jaimes ${ }^{1}$ \\ milton.bermudez@javeriana.edu.co \\ Hugo Alberto Escobar Melo ${ }^{2}$ \\ escobarh@javeriana.edu.co \\ María Caridad García-Cepero ${ }^{3}$ \\ maria.caridad.garcia@gmail.com,maria.caridad@javeriana.edu.co \\ José Guillermo Martínez Rojas ${ }^{4}$ \\ jose.martinez@javeriana.edu.co \\ Eunice Prada Nagay \\ euniceprada@yahoo.com \\ Andrés Samper Arbeláez6 \\ a.samper@javeriana.edu.co
}

(DOI: 10.17230/ricercare.2015.4.6)

1 Milton Eduardo Bermúdez Jaimes. Colombiano. Doctor en Psicología, Desarrollo y Medición y Evaluación, Docente de planta, Pontificia Universidad Javeriana, Facultad de Psicología, Departamento de Psicología.

2 Hugo Alberto Escobar Melo. Colombiano. Psicólogo, Universidad del Valle, Cali. Magíster en Psicología, Pontificia Universidad Javeriana, Bogotá. Docente, Pontificia Universidad Javeriana, Facultad de Psicología, Departamento de Psicología.

3 María Caridad García-Cepero. Psicóloga, Universidad de los Andes, Bogotá; maestra en Educación, desarrollo del talento y superdotación, Universidad de Houston; doctora en Psicología Educativa con énfasis en desarrollo del talento y superdotación, Universidad de Connecticut. Profesora asociada, Pontificia Universidad Javeriana, Facultad de Educación, Departamento de Formación.

4 José Guillermo Martínez Rojas. Colombiano. Licenciado en Filosofía y Teología y magíster en Educación de la Pontificia Universidad Javeriana. Especialista en Legislación Educativa y Procedimientos de la Universidad Autónoma de Colombia. Magíster en Docencia e Investigación Universitaria de la Universidad Sergio Arboleda. Candidato a Doctor en Educación de la Universidad de Barcelona. Afiliación institucional: Pontificia Universidad Javeriana. Docente de planta, Pontificia Universidad Javeriana, Facultad de Educación, Departamento de Formación.

5 Eunice Prada Nagay. Rusa-colombiana. Master en Pedagogía Musical, Profesora asistente de tiempo completo, Pontificia Universidad Javeriana, Departamento de Música.

6 Andrés Samper Arbeláez. Colombiano. Pregrado en música, Université du Québec à Montréal; especialista en Gerencia y Gestión Cultural, Universidad del Rosario; magíster en Educación, Pontificia Universidad Javeriana; candidato a doctor en Educación Musical, London Institute of Education, University College of London. Docente de planta, Pontificia Universidad Javeriana, Departamento de Música. 


\section{Resumen}

Este documento presenta los resultados de un estudio que evaluó el impacto de los procesos de formación musical que se están adelantando en los colegios públicos de Bogotá dentro de la primera fase del proyecto de la alcaldía local 'Jornada escolar 40 horas'. Un equipo interdisciplinar (música, educación y psicología) evaluó una población aproximada de 373 estudiantes pertenecientes a 29 colegios y obtuvo un diagnóstico del el desarrollo de los saberes musicales de los estudiantes; el impacto de los procesos formativos sobre dimensiones no musicales como autoconcepto, autoestima, empatía y motivación; la calidad y el alcance de las estrategias pedagógicas empleadas en las aulas de clase; la percepción sobre el proceso por parte de estudiantes, profesores, padres de familia y directivos de los colegios; el nivel de adecuación de la infraestructura y de los recursos materiales empleados. El producto final del proyecto es una batería de pruebas que permitirá continuar monitoreando a corto y mediano plazo los procesos de educación musical de este programa y su impacto sobre las dimensiones abordadas.

Palabras clave: educación musical pública, evaluación de la educación musical, música y desarrollo psicosocial.

\section{Abstract}

This paper presents the results of a study that evaluated the impact of musical training processes in public schools of Bogotá in the first phase of the local administration's program 'Proyecto Jornada Escolar 40 Horas'. An interdisciplinary team (music, education, and psychology) evaluated a population of approximately 373 students from 29 schools. We obtained a diagnosis of the development of musical knowledge of the students, as well as of the impact of educational processes on non-musical dimensions such as self-concept, self-esteem, empathy and motivation. The quality and scope of teaching strategies used in the classroom, the perception of the process by students, teachers, parents and principals of schools; the level of adequacy of infrastructure and material resources used, were also diagnosed. . The final product of the project is a battery of tests that may be useful for short and medium term monitoring of this public program and its impact on the dimensions addressed throughout the study.

Keywords: Public music education, assessment in music education, music and psychosocial development 


\section{INTRODUCCIÓN}

El presente artículo recoge los resultados de un estudio que tuvo como propósito evaluar, a partir de una mirada mixta (cualitativa y cuantitativa) el despliegue y el impacto de los procesos de formación musical que se están adelantando en los colegios del Distrito Capital de Bogotá que hacen parte de la primera fase del proyecto "Jornada escolar 40 horas"

El proyecto de investigación se planteó con una perspectiva interdisciplinar de los departamentos de Música, Formación y Psicología de la Pontificia Universidad Javeriana con el fin de valorar los siguientes aspectos en una población aproximada de 373 estudiantes pertenecientes a 29 colegios distritales:

- Desarrollo de los saberes musicales de los estudiantes.

- Impacto de los procesos formativos sobre dimensiones no musicales como autoconcepto, autoestima, empatía y motivación.

- Calidad y alcance de las estrategias pedagógicas empleadas en las aulas de clase.

- Percepción sobre el proceso por parte de estudiantes, profesores, padres de familia y directivos de los colegios.

- Nivel de adecuación de la infraestructura y de los recursos materiales empleados.

Dentro de este marco, el producto final del proyecto fue una batería de pruebas validada con la población evaluada que le permite a la Secretaría de Cultura, Recreación y Deporte monitorear a mediano y largo plazo el desarrollo e impacto de los procesos de educación musical en el programa "Jornada extendida 40 horas" en las dimensiones musicales y psicoafectivas de los estudiantes.

7 La administración distrital actual de Bogotá dio inicio a un ambicioso programa de complemento a la formación de los estudiantes del sector público de la ciudad de Bogotá mediante la implementación del proyecto "Jornada escolar 40 horas". En el marco de dicha jornada extendida se les han ofrecido a los estudiantes actividades deportivas y artísticas como complemento a su formación escolar.

\section{JUSTIFICACIÓN}

Dada la pobre presencia de las artes en los currículos escolares, el campo de la educación artística en Bogotá celebró esta iniciativa del gobierno local, que permite saldar de alguna manera los vacíos curriculares que existen en este sentido, pues está cada vez más clara la importancia de los procesos de formación en artes como aspectos fundantes del desarrollo integral de los niños y jóvenes, aportantes de miradas sensibles y críticas frente al mundo, así como del desarrollo de fortalezas en lo actitudinal, lo axiológico y lo cognitivo.

Por lo mismo, asumir de manera responsable esta oportunidad que se abre para la educación artística en la ciudad implicó planear de una manera estructurada los programas y el desarrollo de los mismos. Asimismo, condujo a hacer un seguimiento riguroso y una evaluación constante de los procesos de desarrollo en las distintas áreas de las artes -en este caso del área musical- tanto en sus aspectos disciplinares y pedagógicos como en su impacto sobre el desarrollo psicosocial y afectivo de los niños y jóvenes.

En este sentido, el presente estudio busca generar una línea de base así como un aparato de evaluación multidimensional que permita, desde una perspectiva interdisciplinar, hacer una primera valoración del desarrollo de los procesos musicales en el "Programa 40 horas" y de su impacto psicosocial sobre los niños y jóvenes evaluados.

\section{ESTADO DEL ARTE DE LA INVESTIGACIÓN}

El interés contemporáneo por la educación artística se ha venido haciendo evidente en las políticas públicas de varios países que promulgan la obligatoriedad de la educación artística. En el marco de la implementación de las políticas públicas surge la pregunta por el seguimiento y la evaluación de los procesos y programas de educación artística: ¿cómo evaluar el desarrollo de las artes en la escuela?, ¿cuáles dimensiones deben ser valoradas?, ¿mediante cuáles tipo de mecanismos y procesos? 
Dado que la investigación local en relación con la evaluación de proyectos públicos de formación en educación artística es aún incipiente, un punto de partida importante en el proceso de dar respuesta a dichos interrogantes es la revisión de referentes regionales e internacionales que han recorrido este camino.

Para empezar, una revisión de proyectos de jornada extendida en América Latina permite constatar propuestas de seguimiento y evaluación no de necesidad orientadas hacia el campo de las artes.

En este sentido, la Administración Federal de Servicios Educativos en el Distrito Federal de Ciudad de México (2009) propuso algunas líneas generales de dimensiones que deben tenerse en cuenta al momento de realizar el seguimiento de las actividades pedagógicas del proyecto. En la parte inicial brinda ideas para la organización del tiempo entre áreas y según actividades; se acentúa la importancia de que los docentes sean cualificados de manera idónea y que puedan disponer de espacios propicios (físicos y de tiempo) para la adecuada planeación, reflexión y evaluación académica dentro de dinámicas de trabajo colaborativas al interior de los equipos. El seguimiento general a la implementación del plan se evalúa mediante un sistema de encuestas aplicadas a supervisores, directores, docentes, padres de familia y estudiantes.

Por otra parte, y dentro de una perspectiva de seguimiento cuantitativo, en la provincia argentina de Río Negro, el gobierno propuso, para el monitoreo de la jornada extendida, un seguimiento mediante indicadores que valoran, en el sentido estadístico, el desarrollo de cinco dimensiones:

1. Infraestructura educativa.

2. Equidad en las escuelas.

3. Jornada escolar.

4. Formación y capacitación.

5. Equipos, mobiliarios, textos, recursos pedagógicos, conectividad y TIC.
En el contexto local, un referente importante para la evaluación de la educación pública es el aportado por el Instituto para la Investigación Educativa y el Desarrollo Pedagógico (IDEP), en Bogotá. Pérez Murcia (2007) presenta una revisión crítica de los principales sistemas de indicadores sociales, tanto en el país como en el mundo y reconoce las falencias y bondades de cada uno de ellos. En este marco de referentes se plantea un modelo de indicadores que no solamente mida tendencias en asuntos como la universalidad del acceso, sino también en temas neurálgicos relacionados con derechos sociales fundamentales como la no discriminación o el asunto de la complementariedad entre las distintas dimensiones de lo educativo.

En el campo de lo artístico, esta visión llama la atención, pues no se trata solamente de que el sistema público logre una amplia cobertura en cuanto a la educación en las artes, sino que lo haga de una manera que impacte de manera significativa las subjetividades, las realidades humanas, los contextos y que sea incluyente de la diversidad.

En algunos países se han desarrollado ejercicios importantes de construcción de referentes y estándares especializados que delimitan el tipo de saberes que deben ser desarrollados en cada una de las áreas, lo cual en sí mismo ofrece herramientas e insumos concretos para poner en marcha procesos de monitoreo y seguimiento al desarrollo de los aprendizajes. El modelo estadounidense de educación artística a través de estándares, por ejemplo, propone para el ámbito formal una estructura basada, en lo primordial, en el desarrollo de dos tipos de estándares: de contenido y de logro. Los primeros determinan las líneas de desarrollo transversales en todos los niveles y los segundos determinan los de desarrollo o de logro esperados para cada uno de los mismos o ciclos (Samper Arbeláez, 2011).

En Argentina (Forero, 2011) se promueve una estructura general de educación artística basada en la tríada entre producción, recepción y apreciación, que 
enmarca la experiencia del arte en la escuela y que promueve el desarrollo de las facultades de percepción, interpretación y agencia en el mundo de lo simbólico.

Otro referente importante es el documento producido por la Asociación Colombiana de Facultades de Artes (ACOFARTES) (De la Guardia, Samper Arbeláez y Sarmiento, 2011) en el marco del diseño de las pruebas ICFES-Saber Pro, que plantea una evaluación de las artes desde una mirada que recupera la idea de tal actividad como herramienta de aprendizaje, que implica procesos de monitoreo con múltiples formas y soportes para evaluar.

En este sentido, el documento mencionado en el párrafo anterior plantea las siguientes competencias transversales a los distintos campos de las artes:

- Expresión-creación.

- Percepción.

- Estético-discursivas.

- Técnicas.

- Socioculturales.

Más adelante, el documento aterriza estas competencias en saberes específicos que pueden valorarse mediante pruebas concretas:

- Identificación visual/áurea.

- Reconocimiento de elementos histórico-culturales.

- Teoría.

- Materiales.

- Historia y literatura.

- Nuevas tecnologías.

Sin embargo, el documento hace un llamado a reconocer que las pruebas masivas deben incluir algún tipo de componente que permita valorar otros asuntos claves del desarrollo artístico como la expresividad, la sensibilidad y la creatividad.

En síntesis, se puede decir que un modelo de seguimiento y monitoreo, como el que se buscó construir mediante este proyecto, debe mantener un equilibrio cuidadoso entre cobertura (amplitud de población) y profundidad en la valoración del desarrollo de las aptitudes musicales en sus componentes fundantes (habilidades, conocimientos, expresividad, sensibilidad y creatividad), así como de los aspectos psicoafectivos de los estudiantes valorados.

\section{MARCO TEÓRICO}

\section{Aspectos musicales}

El creciente interés mundial que se percibe en la actualidad por la formación artística tiene que ver de manera especial con una comprensión del impacto que la misma tiene sobre la educación en general y sobre la formación integral de los individuos, así como sobre las comunidades locales y sus culturas. En este marco de ideas, organizaciones como la UNESCO abogan por la promoción en los países de dicho tipo de formación, al reconocer al arte como un "elemento medular de la naturaleza del ser humano y una característica esencial de su intelecto y sus emociones" (Unesco, 2005, p. 1).

En Colombia, el MEN (2010) valora la dimensión artística como una que favorece la posibilidad del estudiante para percibir el mundo que lo rodea de manera sensible y como un espacio para el desarrollo de saberes que pueden ser aplicables en otras áreas del conocimiento; también como un espacio para que el estudiante entre en contacto con los patrimonios culturales universal y local, en el marco de experiencias reflexivas, críticas e incluyentes.

En línea con dicho pensamiento, la pedagoga musical e investigadora argentina Violeta Hemsy de Gainza (2011; 2013) diría que la educación musical es un derecho humano universal, y que debería, por ende, ser accesible de manera gratuita y con calidad para toda la población. Para alcanzar esto, ella propone un modelo de pedagogías musicales abiertas: formas de enseñar situadas en contextos específicos, desarrolladas a partir de las necesidades, rasgos emergentes y recursos disponibles en contextos específicos. Más allá de casar- 
se con prescripciones y recetarios pedagógicos, se trata más bien de lograr prácticas que desde la perspectiva del sentido común, en diálogo con las propuestas metodológicas tradicionales, logren procesos significativos de musicalización en los niños. ${ }^{8}$

Referentes de otros países, tales como el modelo de estándares de Estados Unidos, plantean de manera muy concreta ciertos contenidos claves para los currículos: canto, interpretación, improvisación, composición, lectura, audición, apreciación y relación de la música con otras artes (Samper Arbeláez, 2011). La ley orgánica de educación de España (LOE) hace acento sobre el asunto de la creatividad, la expresividad y la sensibilidad estética, al tiempo que sugiere un trabajo de aula que va más allá de los instrumentos musicales y del canto al proponer el movimiento corporal (Navarro 2012).

En ambos modelos existe un énfasis balanceado y, sin duda, intencionado, entre las dimensiones de producción, recepción, apreciación y comunicación de la música.

\section{Aspectos pedagógicos}

De acuerdo con la ley 115 de 1994 (Congreso de Colombia, 1994), se puede entender por currículo el conjunto de criterios, planes de estudio, programas, metodologías y procesos que contribuyen a la formación integral y a la construcción de la identidad cultural nacional, regional y local, con inclusión también de los recursos humanos, académicos y físicos para poner en práctica las políticas y llevar a cabo el proyecto educativo institucional. En este marco de ideas, el término "diseño curricular" alude a "boceto", "esquema", "plano", es decir, una representación de ideas, acciones y objetos que se constituye en una guía orientadora a la hora de llevar a la práctica el proyecto curricular. El objetivo del desarrollo del currículo es mejorar las escuelas mediante el perfeccionamiento de la enseñanza y el aprendizaje.

De otra parte, el aprendizaje es el resultado de la interacción mutua entre el individuo y la cultura (Martínez Rodríguez, 1999). En este sentido, es importante entender las maneras en que las interacciones entre los diferentes actores sociales (docentes, estudiantes, familia y comunidad) regulan dicho proceso. Desde una mirada sociocultural, los instrumentos de mediación (e.g. el lenguaje, las preguntas, los materiales impresos, los instrumentos musicales) cumplen un papel importante en el proceso de aprendizaje, pues el desarrollo de habilidades y conocimientos, en este caso musicales, está mediado por herramientas y signos culturales (Baquero, 2000; Martínez Rodríguez, 1999). Las acciones que enmarcan estos procesos pasan de ser una secuencia de actividades a ser "acciones mediadas" en el momento en que un agente intencional (docente), organiza la oportunidad de aprendizaje (acciones), de manera tal que medien entre el niño y su comprensión del mundo. Las acciones humanas, desde la perspectiva de Burke (citado por Wertsch, 1999), involucran cinco elementos fundamentales: el acto, la escena, los agentes, la agencia y el propósito. Estos elementos se constituyen en unidades de análisis para abordar el estudio de las acciones docentes y nos permiten entenderlas trascendiendo la actividad programática.

8 Para conocer más a fondo el pensamiento de Violeta Hemsy de Gainza se sugiere consultar los artículos de su autoría disponibles en la página web: http://www.violetadegainza.com.ar 


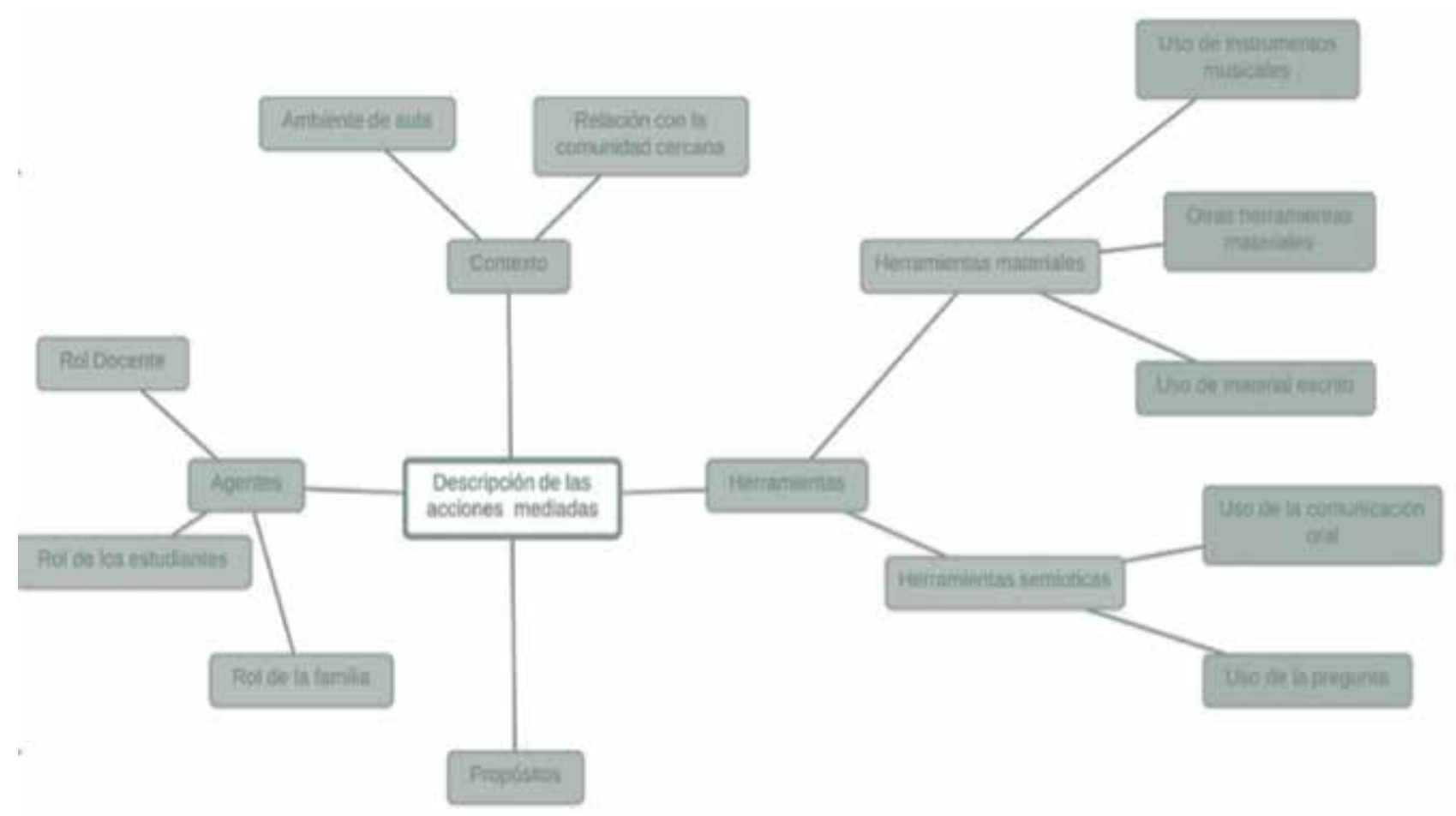

Figura 1. Dimensiones de la acción mediada

Por último, es posible analizar las acciones mediadas desde una perspectiva de la calidad de su gestión, lo cual implicaría, al menos, una aproximación a los niveles de coherencia de las acciones, el de pertinencia de las mismas, si son sostenibles en el tiempo y si son eficaces.

\section{Aspectos psicosociales}

La evidencia empírica que señala el impacto de los programas de formación musical sobre los niños se ha concentrado en tres áreas fundamentales: rendimiento académico, desarrollo cognitivo y dimensiones psicosociales.

En el primer caso, se ha utilizado como referente de evaluación el promedio académico de los niños o se han determinado áreas nodulares como la lectura o las matemáticas para hacer seguimiento (Bhide, Power y Goswani, 2013); en el segundo se han empleado escalas que evalúan múltiples dimensiones tales como atención, memoria, comprensión verbal e inteligencia. En último lugar encontramos los estudios que evalúan tanto los resultados como el impacto que tiene la formación musical en los niños, que consideran que su aporte es mayor tanto en dimensiones personales como contribución al capital social. En esta área se concentra la presente investigación, al indagar sobre las siguientes dimensiones: 


\section{Niños}

- Autoestima: escala de autoestima de Rosenberg

-Autoconcepto: autoconcepto de forma A

-Inventario de bienestar infantil: dimensiones objetivas y subjetivas

\section{Profesores}

-Escala de cmpetencia social

- Cuestionario de fortalezas y debilidades SDQ

\section{Padres de familia}

- Escala de competencia social

-Cuestionario de fortalezas y debilidades SDQ

Figura 2. Dimensiones de la investigación

\section{Objetivos del estudio}

General:

Diseñar un mecanismo de monitoreo y seguimiento a los procesos de formación musical desarrollados en el marco del programa de 40 horas - Jornada extendida de la Alcaldía de Bogotá.

Específicos:

- Evaluar el desarrollo de los saberes musicales de los estudiantes en cuanto a ritmo, voz cantada, reconocimiento auditivo y creatividad.

- Valorar el impacto de los procesos formativos sobre dimensiones no musicales como autoconcepto, autoestima, empatía y motivación.

- Evaluar la pertinencia de las estrategias metodológicas empleadas en los procesos de formación musical.

- Valorar la percepción sobre el proceso por parte de estudiantes, profesores, padres de familia y directivos de los colegios.

- Valorar la pertinencia y el nivel de adecuación de la infraestructura y de los recursos materiales empleados.

- Ofrecer a las Secretarías de Educación y de Cultura una retroalimentación que enriquezca el desarrollo de los procesos de formación musical en la jornada extendida del distrito en cuanto a los contenidos y a los aspectos metodológicos. 


\section{METODOLOGÍA}

El estudio empleó una metodología de carácter mixto que implicó una valoración cuantitativa y cualitativa del impacto del proyecto mediante la utilización de encuestas, entrevistas, grupos focales, observaciones, pruebas de aptitudes musicales e instrumentos de valoración psicométrica.

A fin de tener seguridad sobre la representatividad en el proceso de evaluación fue importante garantizar que la recolección de la muestra fuera aleatoria, es decir, que los sujetos tuvieran las mismas probabilidades de ser escogidos. Por lo tanto, se utilizó un procedimiento probabilístico para seleccionar los individuos que conformaron la muestra definitiva. Se estimó un tamaño de muestra de 373 alumnos (29 colegios) para una población total de 8.922 alumnos.

Los instrumentos diseñados para recoger información se aplicaron de la siguiente manera:

1. Pruebas de aptitudes musicales: con carácter individual; se aplicaron en forma directa en los colegios.

2. Formato de observación pedagógica-música: durante las clases de música en el colegio o en los clanes.

3. Encuesta a docentes-formación: se aplicó de manera virtual.

4. Entrevista a estudiantes-formación, grupos focales: se realizaron in situ, en el mismo lugar en el que se desarrollan las clases.

5. Encuesta para padres: aplicación directa.

6. Instrumento para padres-psicología: se aplicaron de manera telefónica.

7. Instrumento para profesores-piscología: se aplicaron en forma directa en los colegios.

8. Instrumento para estudiantes-psicología: se realizaron in situ, en el mismo lugar en el que se desarrollaron las clases.

Se realizaron reuniones informativas y para la validación de las pruebas con representantes de: Secre- taría de Cultura de Bogotá, IDARTES ${ }^{9}$, directores del programa de 40 horas (OFB ${ }^{10}$ e IDARTES), formadores y organizaciones de la OFB, equipo técnico de la Secretaría de Cultura, auditor del proyecto, equipo de coordinación académica de la Pontificia Universidad Javeriana y equipos de evaluadores por áreas. Al final del estudio se realizó una jornada de socialización de resultados en la que participaron representantes de las instituciones encargadas de la formación musical en el programa de 40 horas, así como rectores y docentes de los colegios evaluados.

\section{RESULTADOS Y CONCLUSIONES GENERALES DEL ESTUDIO}

A continuación se presenta una relación de los principales hallazgo del estudio, de manera discriminada por aptitudes musicales, asuntos pedagógicos y dimensión psicosocial.

En cuanto al desarrollo de aptitudes musicales se llegó a las siguientes conclusiones:

- Se observa de manera evidente un mejor desempeño en la dimensión rítmica en relación con las demás.

- Se observa una relación natural de los estudiantes con el asunto de la canción, lo que puede indicar que está presente dentro de las distintas propuestas.

- Los estudiantes cantan en la mayor parte de las clases con apoyo armónico de guitarra.

- Las entidades se encuentran de manera generalizada en la etapa de iniciación musical.

- Los conceptos musicales han sido en general trabajados de manera contextualizada sobre la música misma; se evidencia una ausencia de ejercicios y conceptualizaciones abstractas relacionadas con la audición y la percepción.

- La prueba de creatividad, que presenta rangos de evaluación más libres, permite evidenciar en los estudiantes una gran capacidad para encontrar recursos diversos, tanto musicales como extramusicales.

9 IDARTES: Instituto Distrital de las Artes.

10 OFB: Orquesta Filarmónica de Bogotá, 
En relación con la dimensión pedagógica, se pudo concluir lo siguiente:

- En general hay un buen desempeño pedagógico por parte de los docentes, evidenciado en su manejo de contenidos, el uso de estrategias didácticas eficientes, la habilidad para adaptarse a las particularidades de los procesos individuales, la escogencia adecuada de contenidos para las clases, el buen manejo de grupo y la habilidad para generar motivación.

- Se encontró una relación directa entre el nivel de formación de los docentes y los de desempeño. A mayor nivel de formación, mejor nivel de desempeño. Resulta, por lo tanto, un asunto importante para considerar.

- No hay garantías de que todas las instituciones cuenten con las herramientas materiales necesarias para cumplir los objetivos pedagógicos.

- Hay dos propósitos diferentes entre los agentes participantes en las acciones mediadas. El primero es enseñar música a los niños para desarrollar en ellos dominios musicales y el segundo es utilizar el espacio de clase como un lugar de esparcimiento para que disfruten.

- Las acciones realizadas fueron percibidas en gran medida como intencionales, lo cual es indicio de la existencia de procesos de planeación y monitoreo, que deben fortalecerse en algunas de las instituciones.

- La relación entre los actores se manifiesta como apropiada en la mayoría de los casos; sin embargo, es importante apoyar aquellas aulas en las que la relación entre estudiantes no son adecuadas, o en las que el docente asume un rol autoritario o permisivo.

- Las bandas criminales y delincuenciales son otro agente de la acción mediada, razón por la cual, es necesario profundizar acerca del papel activo de las mismas en el funcionamiento del programa.

- Es clave que las actividades realizadas puedan relacionarse con los intereses y el contexto de los estudiantes y que, a su vez, se incentive al estudiante para utilizar lo aprendido en otros entornos.
De otra parte, el componente psicosocial de las muestras permitió llegar a los siguientes hallazgos:

- Dado que los instrumentos tuvieron niveles de confiabilidad aceptables, los hallazgos permiten establecer que los resultados son una línea de base importante para el seguimiento y el acompañamiento posterior de la información del monitoreo del programa.

- Con respecto a las dimensiones que indican fortalezas en los alumnos, tales como sus niveles de autoestima, autoconcepto, comportamiento prosocial y competencia social, se recomienda seguir fomentando el programa pues, sin lugar a dudas, impactará de manera positiva en los desempeños de los niños en este nivel.

- Se deben tomar las precauciones debidas en aquellas dimensiones evaluadas que indican la posible presencia de dificultades o déficit, como, por ejemplo, las relaciones familiares percibidas, los problemas de conducta, las dificultades en la relación con los compañeros, los niveles de hiperactividad y los síntomas emocionales.

- Al revisar los cruces entre resultados de aptitudes musicales y la valoración psicosocial, se encontraron relaciones preliminares entre las diferentes categorías.

Por último, es importante señalar que este estudio permitió construir una línea de base, que nos brinda una "fotografía" o diagnóstico de un momento inicial en el proceso del programa de 40 horas desde los puntos de vista musical, pedagógico y psicosocial. El real desarrollo musical de los estudiantes y la correlación del mismo con las dimensiones psicosociales de los niños serán susceptibles de ser observados solamente mediante futuras aplicaciones de las pruebas, que nos permitirán conocer la evolución de los alumnos en dichas dimensiones. En otras palabras, solamente evaluando de nuevo a un estudiante podremos saber cómo han mejorado (o no) su afinación, su audición y su ritmo y si esto ha tenido (o no) un impacto positivo sobre su desarrollo psicosocial, con respecto a los resultados obtenidos con este mismo niño en la prueba 
diagnóstica realizada por el presente proyecto. De igual manera, solamente futuras aplicaciones de las pruebas nos permitirán saber si se ha dado una evolución desde el punto de vista del desempeño pedagógico de los maestros, así como de la adecuación de los recursos físicos (salones, instrumentos, etc.) para el correcto desarrollo de las clases.

Aun así, y solo disponiendo de esta primera aplicación de las pruebas, fue posible encontrar, con una submuestra de 146 niños, las siguientes asociaciones significativas positivas (medidas por los respectivos coeficientes de correlación) entre:

- Conducta prosocial y voz cantada y afinada: 0,17.

- Autoconcepto global y voz cantada y afinada: 0,33.

Esto quiere decir que entre los niños evaluados, aquellos que tenían una mejor afinación en la voz cantada mostraban al mismo tiempo una mejor conducta prosocial y un mejor autoconcepto global. Esta correlación puede ser producto de la experiencia musical brindada por el programa, o puede estar asociada con aspectos extraescolares, como, por ejemplo, las características positivas del entorno familiar: es posible que en un entorno familiar en el que se tiene acceso a referentes musicales ricos, exista también un nivel de trato humano adecuado para generar una buena conducta prosocial y un buen autoconcepto en los niños. Dicho de otro modo, pudimos observar una correlación positiva entre afinación y conducta y autoconcepto, pero solo podremos saber si la misma se origina en realidad en la formación musical más adelante, cuando en futuras pruebas estemos en capacidad de constatar que aquellos niños que mejoren su afinación también lo hagan en su autoconcepto y su conducta prosocial, de modo independiente del tipo de entorno familiar en el que vivan.

Más allá del ámbito de aplicación (público o privado) de este tipo de investigaciones, el camino sigue abierto para que el medio académico continúe indagando de manera sistemática sobre la relación entre formación musical y bienestar integral o desarrollo humano. Se trata de una relación esgrimida en muy alta medida por el sector de la educación artística, pero que aún no dispone de suficiente producción investigativa local que dé cuenta de su evidencia empírica.

\section{REFERENCIAS}

Administración Federal de Servicios Educativos en el Distrito Federal, Secretaría de Educación Pública, SEP, México (2009). Libro blanco del programa Escuelas de Jornada Ampliada 2009-2012. México: Administración Federal de Servicios Educativos en el Distrito Federal, Secretaría de Educación Pública. http://www2.sepdf.gob.mx/ley_transparencia/archivos/jornada_ampliada_2012_2.pdf

Bhide, A., Power, A., \& Goswami, U. (2013). A rhythmic musical intervention for poor readers: a comparison of efficacy with a letter-based intervention. Mind, Brain, and Education, 7(2), 113-123.

Congreso de Colombia (1994). Ley 115, de 8 de febrero de 1994, por la cual se expide la ley general de educación. Bogotá: Congreso de Colombia. http:// www.mineducacion.gov.co/1621/articles-85906_archivo_pdf.pdf

De la Guardia, G., Samper Arbeláez, A., y Sarmiento, M. (2011, enero). Pruebas Saber Pro-ICFES: competencias profesionales para las artes. Bogotá: ACOFARTES.

Forero, P. (2011). "La educación artística en el sistema educativo nacional", un documento que aporta los parámetros actuales para la construcción de un modelo educativo para la enseñanza de las artes en Argentina. Bogotá.

Hemsy de Gainza, V. (2011, enero-junio). Educación musical siglo XXI: problemáticas contemporáneas. Revista da Abem (Associação Brasileira de Educação Musical), 19(25). http://www.violetadegainza. com.ar/2012/05/trabajo-publicado-en-la-revistaabem-asociacion-brasilera-de-educacion-musical/

Hemsy de Gainza, V. (2013). Conferencia inaugural. FLADEM. 
Kalliopuska, M., \& Tiitinen, U. (1991). Influence of two developmental programmes on the empathy and prosociability of preschool children. Perceptual and Motor Skills, 72(1), 323-328.

Martínez Rodríguez, M. Á. (1999). El enfoque sociocultural en el estudio del desarrollo de la educación. REDIE. Revista Electrónica de Investigación Educativa, 1(1), 18, Recuperado el 20 de agosto de http:// redie.ens.uabc.mx/vol1no1/contenido-mtzrod.html

Ministerio de Educación Nacional, MEN (2010). Orientaciones pedagógicas para la educación artística en básica y media. Bogotá: MEN. http://www.mineducacion.gov.co/1621/articles340033_archivo_pdf_ Orientaciones_Edu_Artistica_Basica_Media.pdf

Navarro, J. S. (2012). La educación artística infantil en España según la LOGSE y la LOE. Documento de consulta. Bogotá: Pontificia Universidad Javeriana.

Pérez Murcia, L. E. (2007). ¿Cómo medir el cumplimiento de los derechos sociales? Un modelo de indicadores para monitorear y evaluar el disfrute del derecho a la educación. En Pérez Murcia, L. E., Uprimny Yepes, R., y Rodríguez Garavito, C. (Eds.). Los derechos sociales en serio: hacia un diálogo entre derechos y políticas públicas, pp. 131-177. Bogotá: IDEP. http:// www.corteidh.or.cr/tablas/26466.pdf

Samper Arbeláez, A. (2011). El sistema estadounidense de estándares para la enseñanza de las artes. Bogotá.

UNESCO (2005). Educación artística. http://www. unesco.org/bpi/pdf/memobpi57_artseducation_es.pdf

Wertsch, J. (1999). La mente en acción. Buenos Aires: AIQUE. 